\title{
Formation of Blood Droplets: Influence of the Plasma Proteins
}

\author{
Shantimoy Kar, ${ }^{\dagger, \|}$ Aritra Kar, ${ }^{\ddagger} \perp$ Kaustav Chaudhury, ${ }^{\ddagger} \# \odot$ Tapas Kumar Maiti, ${ }^{\S}$ \\ and Suman Chakraborty*,+0
}

${ }^{\dagger}$ Advanced Technology Development Centre, ${ }^{\ddagger}$ Department of Mechanical Engineering and ${ }^{\S}$ Department of Biotechnology, Indian Institute of Technology Kharagpur, Kharagpur 721302, India

\section{Supporting Information}

ABSTRACT: Blood is a complex multiphase fluid exhibiting pronounced shear-thinning and viscoelastic behavior. By studying the formation of blood droplets through simple dripping, we observe blood-drop detachment following a neck formation and subsequent thinning until breakup, similar to that of other liquids. Our experimental findings reveal that it exhibits two distinct modes of neck evolution characteristics; one mode corresponds to incessant collapsing of the liquid neck, whereas the other mode correlates thinning of an extended long thread leading to the breakup. We show that the two modes of neck evolution closely follow the theory of pinch-off for shear-thinning and viscoelastic fluids independent of hematocrit concentration in the range of healthy individuals.

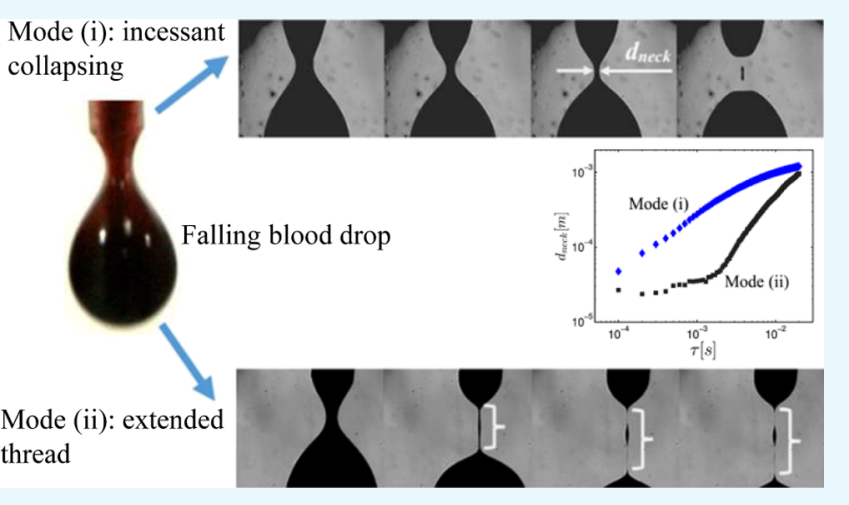
Furthermore, we observe that the relaxation time scales are very similar to that of plasma; this explains the key role of plasma proteins to blood rheology. We envision that our results are likely to bear far-reaching implications in understanding the contribution of plasma proteins to the rheology of blood and theory of drop formation of complex non-Newtonian fluids.

\section{INTRODUCTION}

Understanding the drop formation characteristics for different fluids, including both Newtonian and non-Newtonian, has been realized over the last few decades. ${ }^{1-6}$ Formation of a neck and its subsequent thinning until breakup commonly earmarks the course of droplet detachment in a wide range of scenarios. In complex fluids, intriguing morphological characteristics can be observed. ${ }^{7-11}$ Despite rigorous efforts that have been made toward conceptualizing the droplet detachment processes for fluids with various levels of complexities, common consensus on the physics of blood-drop detachment is yet to be clearly understood. Blood is known to be a heterogeneous suspension of many different cells, proteins, and other small bioanalytes. This leads to intriguing fluidic behavior of blood at different flow conditions, attributed to cellular interactions, hematocrit, plasma chemistry, and so forth. ${ }^{12-15}$ Because of the presence of live biological entities within blood, the interactions are far from being trivial, ${ }^{16-20}$ as compared to the entities suspended in other known complex polymeric fluids. There are indeed experimental evidences, which show that even the blood plasma has an elastic property, because of the interactions of the plasma proteins, which play a dominant role during neck thinning and breakup in elongational flows. ${ }^{12,13}$

The entire process from the neck instigation to the droplet detachment during the breakup event is known as the pinch-off process. From a quantitative perspective, the evolution of the neck diameter, $d_{\text {neck }}$ of the droplet as a function of the time remaining to droplet detachment, $\tau$, is the key focus in the observation of any pinch-off process. Fluidic complexities enter into the picture and an interplay of the capillary, viscous, and inertial forces decide the relationship between $d_{\text {neck }}$ and $\tau$. The phenomenon has been extensively studied for the case of Newtonian fluids. ${ }^{1,2,21,22}$ However, the pinch-off of nonNewtonian fluids depends strongly on respective rheological characteristics and thus makes the pinch-off dynamics more interesting. ${ }^{4,5,8,10,23-25}$ Orientation, clustering, and deformation of the suspended moieties in the blood sample, as well as a combined viscous and elastic nature of the same, impart several complexities toward analyzing blood at pinch. This may be further complicated by the age and health of the concerned subject. Therefore, it necessitates a comprehensive investigation of the physical features occurring over the neck region during blood-drop formation, with an interplay of the diverse and complex rheological features.

Here, we present the flow physics of blood pinch-off, through simple experiments of dripping pendent drop from a tube. We observe two distinct modes of pinch-off, which closely follow the breakup dynamics of shear-thinning and viscoelastic fluids. Fundamentally, these originate from the intricate dynamics of the included blood cells and proteins that are suspended in the plasma matrix. The extended mode of breakup of blood is very similar to that of plasma solution, and

Received: June 7, 2018

Accepted: August 29, 2018

Published: September 11, 2018 
the relaxation time scales are of the same order. These results can be of particular significance to develop theoretical understandings for the formation of blood droplets and their correlation with blood rheology.

\section{RESULTS AND DISCUSSION}

Qualitative and quantitative depiction of the pinch-off characteristics of a representative human blood sample is shown in Figure 1. Two distinct modes of pinch-off can be

(i) Breakup by incessant collapsing of neck
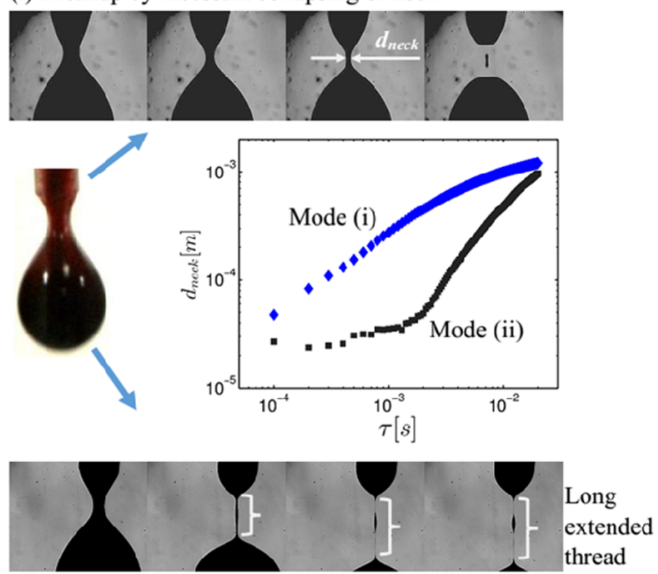

(ii) Breakup by extension of long thread

Figure 1. Pinch-off of a falling pendent drop of blood from tube. Two modes of pinch-off, as observed in the present experiments, are shown: (i) incessant neck collapsing mode (shown for HCT 41.0\%) and (ii) extended thread breakup mode (shown for HCT 39.5\%). Dynamic visualization of the pinch-off processes are provided in the Supporting Information (Movie and corresponding documentation). Corresponding quantitative depiction is provided through the evolution of the neck diameter $d_{\text {neck }}$ in the time remaining to pinchoff $\tau$. The definition of $d_{\text {neck }}$ is also provided in the figure for comprehension.

identified: (i) incessant neck collapsing mode and (ii) extended thread breakup mode. It is worth mentioning that there are stringent dynamic differences, as we shall see. To appreciate this principle, we provide the Supporting Information Movie for comprehensive visualization. Toward quantitative assessment, we present the $d_{\text {neck }}$ evolution as a function of $\tau$, for both the modes mentioned above. Our analysis is focused over $d_{\text {neck }} \approx 10^{-4}$ to $10^{-5} \mathrm{~m}$ (which is also the minimum achievable length scale by using our experimental setup). Studies have shown that the final stage for thinning of complex fluids, even with blood plasma, resides over the neck diameter around $10^{-4}$ to $10^{-5} \mathrm{~m}$. Thus, we specifically analyze the thinning behavior over this regime.

Mode (i) corresponds to the formation of a neck region, which keeps on collapsing until pinch-off and droplet detachment. From the $d_{\text {neck }}-\tau$ data, shown in log-log plot in Figure 1, one can appreciate a power law-type relation $d_{\text {neck }}$ $\propto \tau^{\alpha}$ (with $\alpha$ being the power law exponent) for mode (i) breakup near the point of droplet detachment (evident from nearly linear graph in $\log -\log$ plot). For Newtonian fluids, $\alpha$ is typically either $2 / 3$ or 1 depending on whether the near-pinchoff behavior corresponds to potential flow theory $(\alpha=2 / 3)$ or inertial-viscous flow theory $(\alpha=1){ }^{2}$ For non-Newtonian fluids, however, usually $\alpha=n$, where $n$ is the power law index, whereas the rheological behavior is modeled as power law fluid. ${ }^{4,24}$ For blood pinch-off we also note $\alpha=n$ for the mode (i) pinch-off behavior, as we shall see.

It is important to note that the breakup of viscoelastic fluids is predominantly elongational during the later stage of pinchoff. Under such circumstances, long extension of fluid neck can be observed prior to breakup, for fluids having flexible higher molecular weight polymers. ${ }^{8}$ The polymers undergo stretching in the elongational flow at the later stage of pinch-off and subsequently lead to exponential thinning of the neck. ${ }^{8}$ This is pertaining to the viscoelastic rheology of the complex fluid. In our experiment with human blood, the mode (ii) breakup corresponds to such behavior. Thus, it seems that the flexible proteins and polymers suspended in blood play a vital role during blood pinch-off.

It is worth mentioning that the rheology of the fluid plays a pivotal role in deciding the near-pinch-off behavior. ${ }^{11,26-31}$ However, with the existence of different neck-thinning behavior in blood, as shown in Figure 1, a question seems obvious: what rheological behavior of blood should be considered near pinch-off? The abundant presence of red blood cells (RBCs) pertains to shear thinning rheology of blood, which is often described through power law model. The plasma matrix, however, has interesting rheological characteristics. In shear flow, it exhibits Newtonian behavior; in elongational flow, such as drop breakup scenario, plasma can exhibit viscoelastic behavior. ${ }^{12}$ From our experiments it is evident that even in elongational flow during pinch-off, blood can exhibit either shear thinning or viscoelastic behavior.

It is well known that blood exhibits a combination of shear thinning and viscoelastic behavior. ${ }^{15,20,32,33}$ RBCs form clusters at low shear rates, known as rouleaux. ${ }^{19}$ Plasma proteins, especially fibrinogen, plays a vital role in the formation of these aggregates. The shear-thinning behavior of blood is a consequence of this aggregation behavior leading to higher viscosities at low shear rates. However, at moderate to high shear rates $\left(>10 \mathrm{~s}^{-1}\right)$, the rouleaux structures start to break down, thereby decreasing viscosity and exhibiting a shearthinning behavior. ${ }^{34}$ It is believed that these rouleaux structures can be stretched and deformed, which gives an elastic behavior to the blood. Nevertheless, at shear rates close to $10 \mathrm{~s}^{-1}$ or less, blood shows a more prominent viscous behavior than an elastic behavior.

As the shear rate increases above $100 \mathrm{~s}^{-1}$, the elastic behavior of blood starts to become much more prominent. In measurements of blood rheology under oscillatory shear, the storage modulus and loss modulus quantify the elastic and viscous behavior of fluid at different imposed strain rates. Several experiments performed on blood show a crossover point, indicating the storage modulus to dominate over the loss modulus at increasing shear rates. ${ }^{15,20,33}$ This behavior is counter-intuitive at first glance as the rouleaux structures start to break down at high shear rates, which is the prime reason for elasticity of blood at low shear rates. However, as the clusters break down, they expose to the plasma proteins in the matrix, which indeed possess elastic properties. This has been reported by several research groups, although our experimental findings confirm the dominating elastic behavior of blood at high shear rates. ${ }^{15,20,33}$

Plasma matrix contains several proteins that are essential for the normal functioning of human life. The most substantial plasma proteins in blood are albumin, globulin, and fibrinogen $\sim 55,38$, and $7 \%$, respectively, for a healthy human blood. ${ }^{19}$ Among these three proteins, the viscoelastic properties of 
(a)

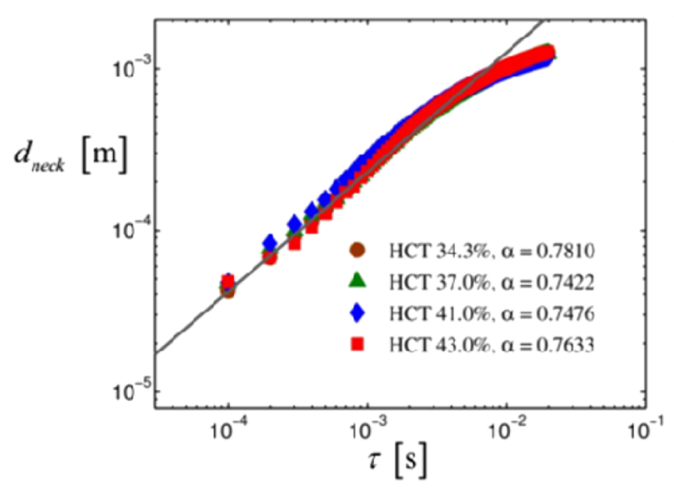

$-d_{\text {neck }} \sim \tau^{0.74}$ Viscous behavior, lcading to incessant collapsing of neck (b)

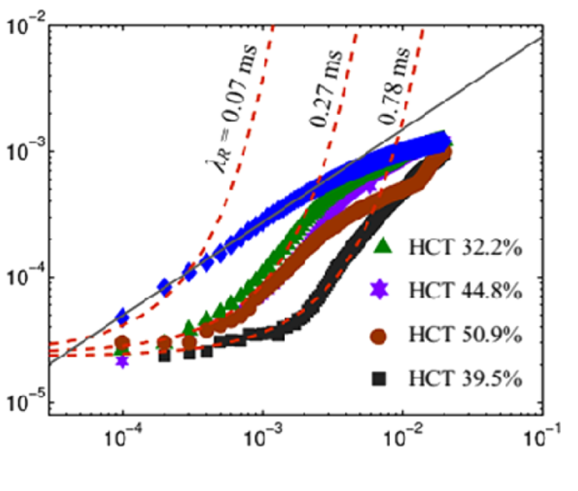

Figure 2. Evolution of neck diameter $d_{\text {neck }}$ in time remaining to pinch off, $\tau$, at various HCT levels: (a) incessant neck collapsing and (b) extensional mode of breakup. An incessant neck-collapsing mode is also presented in (b) for comparison. Accordingly, the experimental observations of the neck thinning are corroborated with scaling laws $d_{\text {neck }} \approx \tau^{0.74}$ (solid lines) and $d_{\text {neck }} \approx \exp \left(\tau / 3 \lambda_{\mathrm{R}}\right)$ (dashed lines). Estimated values of shape relaxation time scales, $\lambda_{\mathrm{R}}$ based on the best fit of the experimental data, are indicated against each exponential observation. The experimental data points are represented as the best fit of the averaged results. Figure S1 in the Supporting Information delineates the experimental uncertainties for each of the experimental data sets (which were averaged over four experimental data sets). In the main article, we have not intentionally added the error bars for better clarity and representation of the figure.

fibrinogen are well known. ${ }^{18,35}$ Under significant forces, as rouleaux structures break down at high shear rates, the fibrinogen structure gets unfolded and exposed in the plasma matrix; this essentially triggers elastic extension of plasma under high shear rates.

An estimate for the relaxation can be given as $\lambda_{\mathrm{R}}=2 \pi \mu_{\text {eff }} a_{\mathrm{p}} /$ $k_{\mathrm{s}}$, referring to the "bead-spring-dumbbell" model. ${ }^{36}$ The model considers a dumbbell-like situation where two spheres of radius, $a_{\mathrm{p}}$, are separated by a distance. The spheres are acted upon by drag force because of fluid flow having effective viscosity, $\mu_{\text {eff }}$ which tries to extend the length. However, a spring-like recoiling force with stiffness $k_{\mathrm{s}}$ tries to resist such elongation. It is important to note that the conceptual paradigm based on the bead spring model is a bit simplistic for a complex multiphase fluid blood. However, we refer to the bead spring model to have an estimate of the relaxation time scales because of the suspended proteins in the plasma matrix.

For our case, the radius of the spheres, $a_{\mathrm{p}}$, can be estimated as $40 \mathrm{~nm},{ }^{35}$ and the spring stiffness, $k_{\mathrm{s}}$, is $\sim 4.5 \mathrm{pN} / \mathrm{nm} .{ }^{13}$ The effective viscosity can be estimated as the elongational viscosity of blood. It was reported in rheological measurements that it is about 20-45 Pa s. ${ }^{13}$ Assuming an intermediate value of $30 \mathrm{~Pa} \mathrm{~s}$ for elongational viscosity of the flow, the current model predicts a relaxation time $\lambda_{\mathrm{R}} \approx 1.67 \mathrm{~ms}$, which closely corroborates our experimental findings. The measured relaxation time scales provide a quantification of the linear viscoelastic properties of plasma proteins suspended in an elongational flow.

Flow field during pinch-off is governed by the momentum balance equation $\rho \partial \mathbf{u} / \partial t+\rho \mathbf{u} \cdot \nabla \mathbf{u}=-\nabla p+\nabla \cdot \boldsymbol{\sigma}$ supplemented with divergence free condition of $\mathbf{u}$ pertaining to incompressibility. The parameters include $\rho$, density; $t$, time; $\mathbf{u}$, velocity vector; $p$, pressure; and $\sigma$, the deviatoric stress tensor. The deviatoric stress tensor, $\sigma$, within the material can be considered to be composed of $\sigma=\sigma_{\text {visc }}+\sigma_{\mathrm{el}}$ viscous $\sigma_{\text {visc }}$ and elastic $\boldsymbol{\sigma}_{\mathrm{el}}$ parts.
Under asymptotic limits close to pinch-off, the axial component of the velocity vector $\mathbf{u}$ is predominant in the neck, leading to uniaxial diverging flow near the neck region. ${ }^{1}$ In the context of drop formation, this is known as the slender jet approximation, which is essentially a one-dimensional formulation. ${ }^{1}$ It is worthwhile to note that the gravity forces are not included in the momentum balance because of the strong gradients near the singular point of pinch-off, which result in the capillary, inertial, and viscous forces to be much stronger. The driving factor for the fluid flow is the capillary pressure gradient, which is scaled as $\nabla p \approx \gamma\left(d_{\text {neck }} z\right)^{-1}$, with $\gamma$ being the coefficient surface tension and $z$ denoting the axial coordinate. The formulation assumes neck curvature scaling as $d_{\text {neck }}{ }^{-1}$, leading to a capillary pressure $p \approx \gamma d_{\text {neck }}^{-1}$.

In the mode (ii) breakup, we can expect the dominance of elastic effect over the viscous effect. The time-dependent elastic stress can be given as ${ }^{8} \sigma_{\mathrm{el}}=\sigma_{0} \exp \left(t / 3 \lambda_{\mathrm{R}}\right)$ during late times $\left(t \gg \lambda_{\mathrm{R}}\right)$, described by a balance between the elastic and capillary forces, with $\sigma_{0}$ being the stress at $t=0$ (constant) and $\lambda_{\mathrm{R}}$ being the relaxation time. In terms of the time remaining to pinch off, $\tau=t_{\mathrm{p}}-t$ (with $t_{\mathrm{p}}$ being the time of pinch-off), the relation reads as $\left(\sigma_{\mathrm{el}}\right)_{z z}=\beta \exp \left(-\tau / 3 \lambda_{\mathrm{R}}\right)$, with $\beta$ being a constant. The balance of elastic and capillary forces leads to an exponential evolution, $d_{\text {neck }} \approx \exp \left(\tau / 3 \lambda_{\mathrm{R}}\right)$. This is typical for viscoelastic pinch-off and has been observed for several polymer solutions. ${ }^{8,12}$ Nevertheless, it should be noted that the exponential regime gives way to other regimes at smaller time scales, which lead to breakup of the neck.

In mode (i) breakup, however, the deviatoric stress is dominated by the viscous stresses. One simple mathematical depiction of the constitutive behavior of blood is the empirical power law formulation that generalizes the Newton's law of viscosity to the form: ${ }^{17} \boldsymbol{\sigma}_{\text {visc }}=m \mathbf{E}^{n}$, where $\mathbf{E}=\frac{1}{2}\left[\nabla \mathbf{u}+(\nabla \mathbf{u})^{\mathrm{T}}\right]$ is a generalized rate of strain dyadic, and $m$ and $n$ are the flow consistency and the flow behavior indices, respectively. 


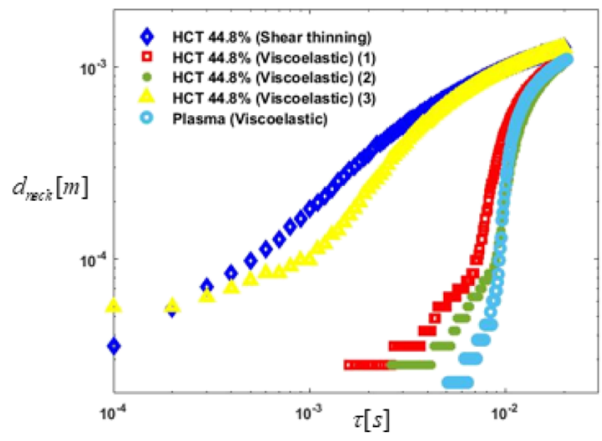

(a)

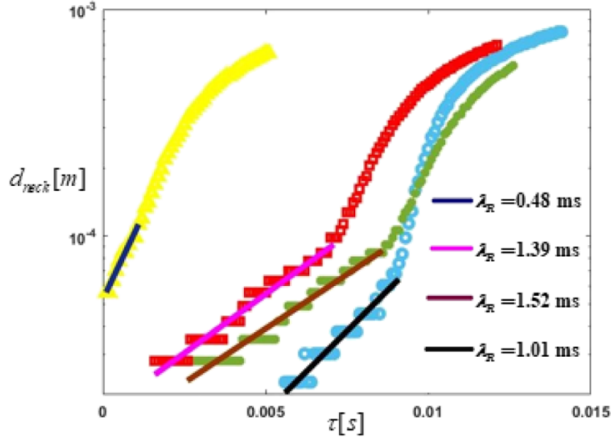

(b)

Figure 3. Evolution of neck diameter, $d_{\text {neck }}$ in time remaining to pinch-off, $\tau$, for the same blood sample and a comparison with its plasma: (a) the plot on the left shows different modes of breakup with varied relaxation times for the same blood sample with HCT 44.8\%. A comparison with the plasma of the same blood sample shows that the extended mode of breakup of blood is similar to the breakup of its plasma solution. (b) The plot on the right shows the same data from (a) on a semilog plot. The straight lines capture the exponential thinning regimes for extended-mode breakup. It is observed at the end of rapid elastic stretching of blood, which is consistent with the literature. The material relaxation time scale, $\lambda_{\mathrm{R}}$, is calculated from the slope of the straight line.

Using the power law formulation, it follows that $\nabla \cdot \boldsymbol{\sigma}_{\text {visc }} \approx$ $m u_{z}^{n} / z^{n+1}$. In asymptotic time scales of breakup, the scaling laws can assume several transient regimes depending on the relative importance of the viscous and inertial forces both within the fluid and the fluid surrounding the breakup. ${ }^{1,21}$ In the case of blood pinch-off, we assume that the viscous forces are relevant throughout the pinch-off, giving rise to asymptotic regimes, where viscosity plays a dominant role. Hence, a balance between pressure gradient and the viscous forces, $\nabla p$ $\approx \nabla \cdot \boldsymbol{\sigma}_{\text {visc }}$ is adequate, and simplifying it in terms of neck diameter, we obtain $d_{\text {neck }} \approx\left(z / u_{z}\right)^{n}$. The temporal characteristics can be directly obtained from balancing the inertial terms, $\rho \partial \mathbf{u} / \partial t \approx \rho \mathbf{u} \cdot \nabla \mathbf{u}$, which leads to $u_{z} \approx z / \tau$. Thus, the neck evolution characteristics in the viscous regime can be given as $d_{\text {neck }} \approx \tau^{n}$, depicting a power law-type behavior. This has also been observed for several shear-thinning fluids. ${ }^{3,21,24}$

The evolution of neck diameter, $d_{\text {neck }}$ in the time remaining to pinch-off, $\tau$, for different blood samples considered in our study, is shown in Figure 2. The results pertain to both (i) dominant viscous behavior leading to incessant neck collapsing (Figure 2a) and (ii) dominant elastic behavior leading to neck extension and breakup (Figure $2 \mathrm{~b}$ ). Considering the scaling arguments, we expect power law-type behavior and exponential neck thinning in the later stages of the corresponding modes of breakup, respectively. The experimental results are thus corroborated with the corresponding scaling laws. The subsequent power law exponents and relaxation time scales are obtained from the experimental and theoretical corroborations.

Values of $\alpha$ corresponding to different blood sample are indicated in Figure 2a. Specifically, $\alpha$ lies around a mean value of $0.74 \pm 0.04266$. Hence, we observe an almost universal scaling behavior for a range of hematocrit from healthy blood donors. In Figure 2a, therefore, we present the theoretical approximation $d_{\text {neck }} \approx \tau^{n}$ with $n=0.74$. We measure the shear viscosity of different blood samples using a Brookfield viscometer. ${ }^{14}$ Subsequently, the experimental data were fitted following a power law consideration with $n=0.74 \pm 0.04266$. Human blood samples collected for our study are pertinent to healthy individuals; thus, $n=0.70-0.78$. The close convergence of the experimental data with the theoretical arguments is noteworthy from the figure, thus validating the scaling arguments.
In Figure 2b, we show the neck evolutions corresponding to the elastic-type extended thread breakup mode characterized by the scaling law $d_{\text {neck }} \approx \exp \left(\tau / 3 \lambda_{\mathrm{R}}\right)$. For the sake of better comprehension, in the figure we also present the sample data corresponding to pinch-off by incessant neck collapsing. The extended mode of breakup is shown for healthy blood samples. It is interesting to note that the exponential thinning with long extended thread formation is prominent for $\mathrm{O}\left(\lambda_{\mathrm{R}}\right)>10^{-1} \mathrm{~ms}$. The elastic-type extension becomes prominent around or before $20 \mathrm{~ms}$ and the relaxation time scales are of the order of $1 \mathrm{~ms}$.

The study of drop formation can be correlated with various non-dimensional parameters, which relate the interplay of the capillary, viscous, and inertial forces. For viscoelastic fluids, the elastic forces become significant, generating relevant parameters related to relaxation times of the fluid. ${ }^{31,37-40}$ The elastocapillary number is generally used to compare the relative magnitude of the elastic forces to the viscous forces (which usually is defined for a fluid with constant viscosity). However, because of the shear-thinning nature of blood, its viscosity is dependent on shear rate and hence we adopt the methods in Clasen et al. ${ }^{30}$ to construct the elasto-capillary number. The characteristic capillary thinning velocity for a viscositydominated flow is given by $U_{\eta}=n \Phi^{1 / n}(\gamma / m)^{1 / n} R^{(n-1) / n}$, where $m$ and $n$ are the flow consistency and flow behavior indices, respectively. ${ }^{30}$ Here, $\Phi$ is a prefactor that depends on the value of $n$. The parameters $\gamma$ and $R$ are the surface tension and the radius of the neck, respectively. The capillary thinning velocity for an elasticity-dominated flow is given by $U_{\lambda}=R$ / $3 \lambda_{\mathrm{R}}$.

Using a value of $n=0.74$ and $\Phi$ from ref 30, we obtain $U_{\eta} /$ $U_{\lambda}=(4.83)^{-1} \lambda_{\mathrm{R}}(\gamma / m R)^{1.35}$. Accordingly, the elasto-capillary number for blood pinch-off can be defined as $E_{c}=\lambda_{\mathrm{R}}(\gamma)$ $m R)^{1.35}$. Evidently, Ec $>4.83$ corresponds to $U_{\lambda}<U_{\eta}$, which represents elastic forces to dominate over viscous forces. For healthy individuals, $m \approx 10-20 \mathrm{cP} / \mathrm{s}^{n-1}$ and $\gamma \approx 50-55 \mathrm{mN} /$ $\mathrm{m}$ (from the Supporting Information, Figure S2). Assuming $m$ $=15 \mathrm{cP} / \mathrm{s}^{n-1}$ and $\gamma=52.5 \mathrm{mN} / \mathrm{m}$, the critical radius of transition $(\mathrm{Ec}=4.83)$ for $\lambda=0.78$ and $0.27 \mathrm{~ms}$ are 5.43 and $2.48 \mathrm{~mm}$, respectively. Indeed, the elastic behavior starts to become prominent from the neck radius approximately around the order of $1 \mathrm{~mm}$ (see Figure $2 \mathrm{~b}$ ). As the neck radius reduces further, $E c \gg 4.83$ and the elastic behavior becomes much 
more prominent, leading to a balance between elastic and capillary forces, giving rise to exponential regimes of the evolution of neck diameter.

It is important to note here that we observed both the modes of breakup for all the blood samples irrespective of its hematocrit value. Hence, the occurrence of a specific mode of breakup cannot be distinguished on HCT values. Two modes of droplet breakup are seen to occur in a random manner for any particular blood sample irrespective of HCT. However, the occurrence of extended mode of breakup $(\sim 80 \%)$ is more than that of the neck-collapsing mode $(\sim 20 \%)$. The random variation in breakup can be attributed to the fact that the blood samples are also varied with different parameters like concentration of plasma proteins. However, more investigation is needed to understand the pinch-off behavior of blood samples that deviate from healthy concentrations of the different constituents.

Furthermore, to realize the random occurrence of breakup, we performed experiments on a particular HCT of $44.8 \%$ and compared it with its plasma, which is depicted in Figure 3. Figure 3a shows the occurrence of both modes of breakup and the plasma solution following an extended mode of breakup. Figure $3 \mathrm{~b}$ captures the exponential relaxation regime as a linear evolution. This phenomenon is common to viscoelastic thread breakup and is observed after a rapid stretching of the thread, consistent with our observations. ${ }^{9,10,12}$ The measured plasma relaxation time of $1.01 \mathrm{~ms}$ is close to the reported value of 0.83 $\mathrm{ms}$ by Brust et al. ${ }^{12}$ However, all observed relaxation times of blood breakup were within $2 \mathrm{~ms}$.

It is interesting to note the stochastic phenomenon leading to a random breakup into one of the modes, which reflects the heterogeneity of blood in the small volume trapped in the neck $(\sim 5 \mu \mathrm{L})$. An estimate of the strain rates inside the neck can be obtained from $\dot{\gamma} \approx \partial u_{z} / \partial z \approx 1 / \tau$ (under slender-jet approximation). Indeed, at time scales of $10^{-2} \mathrm{~s}$, where the separation between the two modes occurs, the strain rates are of the order of $100 \mathrm{~s}^{-1}$, where most of the rouleaux structures break down significantly, leading to a discrete flow within the plasma matrix. ${ }^{34}$ Also, on careful observation of the neck during breakup, one can observe the Fahraeus-Lindqvist effect, where the RBCs reside more near the surface of the neck as the shear rates are higher toward the central axis. This leaves a highly dilute suspension of plasma near the center, which drives the neck to extensional mode of breakup under sufficiently high shear rates. Hence, we mostly observe an extended mode of breakup. However, if the local hematocrit concentration in the neck is high because of heterogeneity, a dominant shear-thinning behavior is observed as the kinetics of breakdown of the rouleaux is delayed beyond the small time scales of $10^{-2} \mathrm{~s}$.

Prior to concluding, it is important to discuss the associated uncertainties observed in our experiments. The bias uncertainty arises predominantly because of the limitations in the camera resolution and image-processing algorithms. A measure of the bias uncertainty for neck diameters below $10^{-4}$ $\mathrm{m}$ is $13.07 \%$, representing the upper limit, whereas for neck diameters around $10^{-3} \mathrm{~m}$, it is $0.8 \%$. The total uncertainty is calculated from the processed experimental data, and a typical set of experiments conducted on HCT of $44.8 \%$ gave a maximum of $14.3 \%$ uncertainty for neck diameters around $10^{-4} \mathrm{~m}$ and $1.4 \%$ for neck diameters around $10^{-3} \mathrm{~m}$. For all the conducted experiments, the uncertainty follows a similar trend with a maximum limit of $\sim 20 \%$, whereas the minimum limit is $\sim 1 \%$. Therefore, it is evident that the associated uncertainty is substantial as the blood column approached the lower length scale until breakup. Thus, the measured uncertainties explain the underlying reason of having scattered data for the neckcollapsing breakup approach. It is also worth noting here that the overall uncertainty is dominated by the bias uncertainties because of the limitations in the experimental setup, whereas the precision uncertainties are found to be less significant in our investigations.

\section{CONCLUSIONS}

In summary, blood pinch-off can take place either by incessant neck collapsing or by extended thread breakup. The former mode is characterized by a dominant shear-thinning behavior of blood, leading to a power law-based collapsing of the neck in the time remaining to pinch-off. The later mode has its genesis in the viscoelastic-type extensional relaxation of the blood. The extensional mode pertains to an exponential evolution of neck diameter in the time remaining to pinch off for the length and time scales mentioned within this paper. The measured relaxation times are within $0-2 \mathrm{~ms}$ and the extended mode of breakup is very similar to that of plasma. We strongly believe that fibrinogen plays a significant role in the viscoelastic nature of plasma solution and the calculated relaxation times of the fibrinogen structure was of the same order. However, it is also possible that other plasma proteins and their intermolecular interactions contribute toward the viscoelastic nature and further research needs to be conducted to understand this phenomenon. This can have far-reaching consequences in describing the rheological characteristics of blood under high shear rates. Despite having the rheological diversities, the macroscopic manifestation of the underlying phenomenon is essentially governed by an inhomogeneous distribution of corpuscles in the blood sample at small length scales, which itself evolves with time. A deeper understanding of the underlying morphodynamics, as portrayed in this work, may bear immense contributions to explain the rheological characteristics of blood, laying groundwork for many other advanced studies on blood droplets and its rheology.

\section{MATERIALS AND METHODS}

We conducted experiments on falling pendent blood drop from a tube. Experiments were performed as per the institutional ethical guidelines of the authors (approval no. IIT/SRIC/AR/2012). Informed consents were taken while collecting the blood samples from the volunteers. The collected blood samples were preserved in anti-coagulantcoated vials at around $8-20{ }^{\circ} \mathrm{C}$. The samples were directly used for experiments without any dilution or chemical treatment. The objective was to maintain the physiologically pertinent condition to the extent possible. Subsequently, experiments were executed within $24 \mathrm{~h}$ after collection of the sample.

Essentially, blood was flown through a cylindrical tube to the ambient. Figure 4 schematically shows the present experimental consideration. The blood was flown through a syringe pump (Harvard Apparatus PHD2000) through a tube having diameter of $1.5 \mathrm{~mm}$. One end of the tube was inserted through the top surface of an enclosed glass tube. It is known that the physical subtleties over the characteristic length and time scales, around the neck area during pinch-off, are independent of the flow features at the far regions. ${ }^{2}$ Thus, volume flow rate 


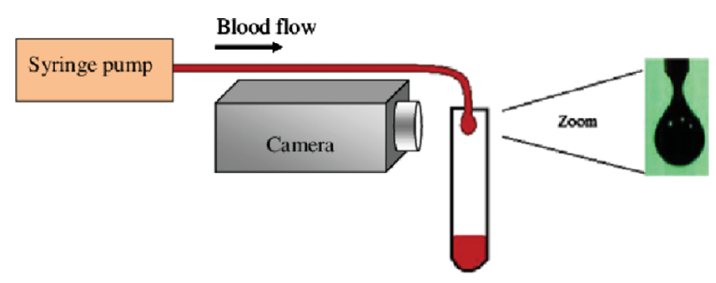

Figure 4. Description of the present experimental considerations: schematic illustration of the experimental setup.

has no influence on the pinch-off dynamics. However, it is usual to employ a constant flow rate so as to ensure an intermittent supply of liquid. ${ }^{2,6}$ Here, we maintained a volume flow rate of $\lambda_{\mathrm{R}}=2 \pi \mu_{\mathrm{eff}} a_{\mathrm{p}} / k_{\mathrm{s}}$, through the syringe pump.

Blood pinched off at the end of the tube in a surrounding medium of air (it can be considered as free-falling blood drop in an enclosed glass tube) (cf. magnified portion of Figure 4). The images of the free-falling blood drop were captured through a high-speed camera (AMETEK V641) at a speed of 10000 frames per second with a resolution of (640 pixels $\times$ 480 pixels). All the captured images were then post-processed through an in-house MATLAB code, which takes care of the calculation of the neck radius using the edge detection method. Repeated experiments were performed, and the average behaviors along with the corresponding standard deviations were reported.

\section{ASSOCIATED CONTENT}

\section{S Supporting Information}

The Supporting Information is available free of charge on the ACS Publications website at DOI: 10.1021/acsomega.8b01279.

The supporting information contains a movie of the different modalities of blood pinch-off observed in our experiment. Figure S1 illustrates the experimental uncertainties for each of the experimental data sets (which were averaged over four experimental data sets). Figure S2 delineates description of different experimental parameters (viscosity, power law exponent $(n)$, and surface tension) (PDF) (AVI)

\section{AUTHOR INFORMATION}

\section{Corresponding Author}

*E-mail: suman@mech.iitkgp.ernet.in (S.C.).

\section{ORCID}

Kaustav Chaudhury: 0000-0002-6595-4695

Suman Chakraborty: 0000-0002-5454-9766

\section{Present Addresses}

"Currently working as Postdoctoral Research Associate in Technische Universitat Darmstadt, Germany 64287.

${ }^{\perp}$ Currently pursuing his doctoral studies at The University of Texas, Austin, United States of America 78705.

"Presently at the Department of Mechanical Engineering, National Institute of Technology Rourkela, Rourkela 769008, India.

\section{Author Contributions}

S.C. conceived and supervised the work. S.K. oversaw the experiments conducted. A.K. and S.K. performed the experiments. K.C. and A.K. performed the theoretical analysis. S.K.,
K.C., and A.K. processed the results. K.C., S.K., A.K., S.C. wrote the paper. T.K.M. and S.C. edited the paper.

Notes

The authors declare no competing financial interest.

\section{ACKNOWLEDGMENTS}

The authors gratefully acknowledge the financial support provided by the Indian Institute of Technology Kharagpur, India (Sanction Letter no.: IIT/SRIC/ATDC/CEM/2013-14/ 118, dated 19.12.2013), and the Royal Academy of Engineering, London, UK (HEPI/1516/27). S.K. acknowledges the fellowship from the Council of Scientific and Industrial Research (CSIR) for carrying out the research work. We sincerely acknowledge Prof. Howard A. Stone, Princeton University, USA, and Prof. Christian Wagner, Universität des Saarlandes, Germany for their valuable comments and suggestions on the paper.

\section{REFERENCES}

(1) Lister, J. R.; Stone, H. A. Capillary Breakup of a Viscous Thread Surrounded by Another Viscous Fluid. Phys. Fluids 1998, 10, 27582764.

(2) Chen, A. U.; Notz, P. K.; Basaran, O. A. Computational and Experimental Analysis of Pinch-Off and Scaling. Phys. Rev. Lett. 2002, 88,174501

(3) Javadi, A.; Eggers, J.; Bonn, D.; Habibi, M.; Ribe, N. M. Delayed Capillary Breakup of Falling Viscous Jets. Phys. Rev. Lett. 2013, 110, 144501.

(4) Aytouna, M.; Paredes, J.; Shahidzadeh-Bonn, N.; Moulinet, S.; Wagner, C.; Amarouchene, Y.; Eggers, J.; Bonn, D. Drop Formation in Non-Newtonian Fluids. Phys. Rev. Lett. 2013, 110, 034501.

(5) Wagner, C.; Amarouchene, Y.; Bonn, D.; Eggers, J. Droplet Detachment and Satellite Bead Formation in Viscoelastic Fluids. Phys. Rev. Lett. 2005, 95, 164504.

(6) Miskin, M. Z.; Jaeger, H. M. Droplet Formation and Scaling in Dense Suspensions. Proc. Natl. Acad. Sci. U.S.A. 2012, 109, 43894394.

(7) Sattler, R.; Wagner, C.; Eggers, J. Blistering Pattern and Formation of Nanofibers in Capillary Thinning of Polymer Solutions. Phys. Rev. Lett. 2008, 100, 164502.

(8) Sattler, R.; Gier, S.; Eggers, J.; Wagner, C. The Final Stages of Capillary Break-up of Polymer Solutions. Phys. Fluids 2012, 24, 023101.

(9) Oliveira, M. S. N.; Yeh, R.; McKinley, G. H. Iterated Stretching, Extensional Rheology and Formation of Beads-on-a-String Structures in Polymer Solutions. J. Non-Newtonian Fluid Mech. 2006, 137, 137148

(10) Clasen, C.; Eggers, J.; Fontelos, M. A.; Li, J.; McKinley, G. H. The Beads-on-String Structure of Viscoelastic Threads. J. Fluid Mech. 2006, 556, 283

(11) Château, J.; Guazzelli, É.; Lhuissier, H. Pinch-off of a Viscous Suspension Thread. J. Fluid Mech. 2018, 852, 178-198.

(12) Brust, M.; Schaefer, C.; Doerr, R.; Pan, L.; Garcia, M.; Arratia, P. E.; Wagner, C. Rheology of Human Blood Plasma: Viscoelastic Versus Newtonian Behavior. Phys. Rev. Lett. 2013, 110, 078305.

(13) Kolbasov, A.; Comiskey, P. M.; Sahu, R. P.; Sinha-Ray, S.; Yarin, A. L.; Sikarwar, B. S.; Kim, S.; Jubery, T. Z.; Attinger, D. Blood Rheology in Shear and Uniaxial Elongation. Rheol. Acta 2016, 55, 901-908.

(14) Kar, S.; Dash, M.; Maiti, T. K.; Chakraborty, S. Effect of Hematocrit on Blood Dynamics on a Compact Disc Platform. Analyst 2015, 140, 1432-1437.

(15) Campo-Deaño, L.; Dullens, R. P. A.; Aarts, D. G. A. L.; Pinho, F. T.; Oliveira, M. S. N. Viscoelasticity of Blood and Viscoelastic Blood Analogues for Use in Polydymethylsiloxane in Vitro Models of the Circulatory System. Biomicrofluidics 2013, 7, 034102. 
(16) Hussain, M. A.; Kar, S.; Puniyani, R. R. Relationship between Power Law Coefficients and Major Blood Constituents Affecting the Whole Blood Viscosity. J. Biosci. 1999, 24, 329-337.

(17) Enderle, J.; Bronzino, J. Introduction to Biomedical Engineering; Academic press, 2012.

(18) Mosesson, M. W. Fibrinogen and Fibrin Structure and Functions. J. Thromb. Haemostasis 2005, 3, 1894-1904.

(19) Baskurt, O. K.; Meiselman, H. J. Blood Rheology and Hemodynamics. Semin. Thromb. Hemostasis 2003, 29, 435-450.

(20) Tomaiuolo, G.; Carciati, A.; Caserta, S.; Guido, S. Blood Linear Viscoelasticity by Small Amplitude Oscillatory Flow. Rheol. Acta 2016, 55, 485-495.

(21) Eggers, J.; Villermaux, E. Physics of Liquid Jets. Rep. Prog. Phys. 2008, 71, 036601.

(22) Eggers, J. Universal Pinching of 3D Axisymmetric Free-Surface Flow. Phys. Rev. Lett. 1993, 71, 3458-3460.

(23) Dinic, J.; Jimenez, L. N.; Sharma, V. Pinch-off Dynamics and Dripping-onto-Substrate (DoS) Rheometry of Complex Fluids. Lab Chip 2017, 17, 460-473.

(24) Doshi, P.; Suryo, R.; Yildirim, O. E.; McKinley, G. H.; Basaran, O. A. Scaling in Pinch-off of Generalized Newtonian Fluids. J. NonNewtonian Fluid Mech. 2003, 113, 1-27.

(25) Tirtaatmadja, V.; McKinley, G. H.; Cooper-White, J. J. Drop Formation and Breakup of Low Viscosity Elastic Fluids: Effects of Molecular Weight and Concentration. Phys. Fluids 2006, 18, 043101.

(26) Dinic, J.; Jimenez, L. N.; Sharma, V. Pinch-off Dynamics and Dripping-onto-Substrate (DoS) Rheometry of Complex Fluids. Lab Chip 2017, 17, 460-473.

(27) Wagoner, B. W.; Thete, S. S.; Basaran, O. A. A New Experimental Method Based on Volume Measurement for Determining Axial Scaling during Breakup of Drops and Liquid Threads. Phys. Fluids 2018, 30, 082102.

(28) Doshi, P.; Basaran, O. A. Self-Similar Pinch-off of Power Law Fluids. Phys. Fluids 2004, 16, 585-593.

(29) Jiang, X. F.; Zhu, C.; Li, H. Z. Bubble Pinch-off in Newtonian and Non-Newtonian Fluids. Chem. Eng. Sci. 2017, 170, 98-104.

(30) Clasen, C.; Phillips, P. M.; Palangetic, L.; Vermant, J. Dispensing of Rheologically Complex Fluids: The Map of Misery. AIChE J. 2012, 58, 3242-3255.

(31) Mckinley, G. H. Visco-Elasto-Capillary Thinning and Break-Up of Complex Fluids; The British Society of Rheology, 2005; pp 1-48.

(32) Thurston, G. B. Viscoelasticity of Human Blood. Biophys. J. 1972, 12, 1205-1217.

(33) Alves, M.; Rocha, C.; Gonçalves, M. P. Study of the Rheological Behaviour of Human Blood Using a Controlled Stress Rheometer. Clin. Hemorheol. Microcirc. 2013, 53, 369-386.

(34) Snabre, P.; Bitbol, M.; Mills, P. Cell Disaggregation Behavior in Shear Flow. Biophys. J. 1987, 51, 795-807.

(35) Zhmurov, A.; Brown, A. E. X.; Litvinov, R. I.; Dima, R. I.; Weisel, J. W.; Barsegov, V. Mechanism of Fibrin(Ogen) Forced Unfolding. Structure 2011, 19, 1615-1624.

(36) Byron Bird, R.; Armstrong, R. C.; Hassager, O. Dynamics of Polymeric Liquids. Volume 1: Fluid Mechanics; Wiley-Interscience Publication, John Wiley \& Sons, 1987.

(37) Clasen, C.; Bico, J.; Entov, V. M.; McKinley, G. H. "Gobbling drops": the jetting-dripping transition in flows of polymer solutions. J. Fluid Mech. 2009, 636, 5.

(38) Clasen, C.; Plog, J. P.; Kulicke, W.-M.; Owens, M.; Macosko, C.; Scriven, L. E.; Verani, M.; McKinley, G. H. How Dilute Are Dilute Solutions in Extensional Flows? J. Rheol. 2006, 50, 849-881.

(39) Campo-Deaño, L.; Clasen, C. The Slow Retraction Method (SRM) for the Determination of Ultra-Short Relaxation Times in Capillary Breakup Extensional Rheometry Experiments. J. NonNewtonian Fluid Mech. 2010, 165, 1688-1699.

(40) Anna, S. L.; McKinley, G. H. Elasto-Capillary Thinning and Breakup of Model Elastic Liquids. J. Rheol. 2001, 45, 115-138. 